\title{
Transplantation de cellules souches in utero chez le mouton
}

\author{
Les nouvelles \\ de ce numéro \\ ont été préparées par : \\ J.-C. Dreyfus \\ A. Kahn
S. Erlinger
F. Cuzin
}

1. Flake AW, Harrison MR, Adzick NS, Zanjani ED. Transplantation of fetal hematopoietic stem cells in utero : the creation of hema-

La plupart des travaux qui visent à guérir les maladies héréditaires actuellement incurables sont axés sur l'introduction de gènes sains dans des cellules atteintes. Dans les maladies les plus fréquentes, thalassémies et drépanocytose, le succès ne peut être qu'à terme, car de difficiles problèmes restent à résoudre. On se tourne donc simultanément vers les méthodes de transplantation. On connaît les succès et les dangers des greffes de moelle, et notamment les difficultés de l'immunosuppression et des réactions du greffon contre l'hôte. Le moyen le plus élégant de résoudre ces difficultés serait d'utiliser, pour le donneur comme pour le receveur, des tissus fotaux dotés de tolérance immunologique. Flake $e t$ al. (San Francisco) ont fait une tentative dans cette voie [1]. Ils se sont intéressés au mouton qui présente plusieurs avantages, en particulier une durée de gestation assez longue (145 jours) pour que des transplantations puissent être tentées au cours de son premier tiers, des caractéristiques immunologiques favorables, avec une tolérance aux greffes allogéniques jusqu'à 67 jours d'âge fœtal au moins. Enfin l'hémoglobine de mouton présente deux allèles normaux de la chaîne $B, A$ et $B$, qui constituent des marqueurs parfaits de la réussite ou du rejet d'une greffe de tissu hématopoïétique. Les auteurs ont préparé des cellules de foie de moutons homozygotes AA d'âge fœtal 35 à 50 jours. Ils ont injecté la suspension par voie intrapéritonéale à huit homozygotes $\mathrm{BB}$ d'ấge fotal 45 à 65 jours. La technique entraîne actuellement une mortalité élevée : quatre survivants seulement, dont trois montraient un chimérisme des cellules sanguines, témoignant du succès de la greffe. Six mois après la naissance, les deux survivants (le troisième est mort d'une infection intercurrente) maintenaient un degré de chimérisme fixe sans aucun signe du syndrome du greffon contre l'hôte. La proportion d'hémoglobine des donneurs était de 15 à $25 \%$ du total et les auteurs pensent qu'on pourrait aisément l'accroître en augmentant les doses injectées.

On peut considérer ce premier résultat comme encourageant ; et il démontre l'intérêt qu'il y a à utiliser des donneurs et des receveurs immunologiquement immatures. Il faudra naturellement répéter l'expérience pour aboutir à une réduction drastique de la mortalité. Ces résultats obtenus, et sans préjuger de considérations éthiques, on pourra songer à d'éventuelles applications cliniques. L'âge des foetus de mouton correspond à un âge gestationnel humain de 18-20 semaines. Grâce à l'emploi des villosités choriales un diagnostic prénatal peut être obtenu beaucoup plus tôt pour la drépanocytose et certaines variétés de thalassémie, mais les dates limite et optimale ne sont pas forcément les mêmes dans les deux espèces. Il est clair qu'avant d'aborder l'homme, des essais sur des primates seront nécessaires. Mais les résultats obtenus permettent d'envisager dès maintenant la production de cellules chimériques par injection de cellules souches au fœetus, comme une chance future d'éviter l'avortement chez les fotus atteints de maladies congénitales du système hématopoïétique.

J.-C. D. 